\title{
On a 1, 2 Conjecture
}

\author{
Jakub Przybyło f| and Mariusz Woźniak|t \\ AGH University of Science and Technology, Al. Mickiewicza 30, 30-059 Kraków, Poland
}

received February 12, 2008, accepted February 3, 2010.

Let us assign positive integers to the edges and vertices of a simple graph $G$. As a result we obtain a vertex-colouring of $G$ with integers, where a vertex colour is simply a sum of the weight assigned to the vertex itself and the weights of its incident edges. Can we obtain a proper colouring using only weights 1 and 2 for an arbitrary $G$ ?

We give a positive answer when $G$ is a 3-colourable, complete or 4-regular graph. We also show that it is enough to use weights from 1 to 11 , as well as from 1 to $\left\lfloor\frac{\chi(G)}{2}\right\rfloor+1$, for an arbitrary graph $G$.

Keywords: neighbour-distinguishing total-weighting, irregularity strength

\section{Introduction}

A $k$-total-weighting of a simple graph $G$ is an assignment of an integer weight, $w(e), w(v) \in\{1, \ldots, k\}$ to each edge $e$ and each vertex $v$ of $G$. The $k$-total-weighting is neighbour-distinguishing (or vertex colouring, see (1)) if for every edge $u v, w(u)+\sum_{e \ni u} w(e) \neq w(v)+\sum_{e \ni v} w(e)$. If it exists, we say that $G$ permits a neighbour-distinguishing $k$-total-weighting. The smallest $k$ for which $G$ permits a neighbour-distinguishing $k$-total-weighting we denote by $\tau(G)$.

A similar parameter, but in the case of an edge (not total) weighting was introduced and studied in (8) by Karoński, Łuczak and Thomason. They conjectured that every simple connected graph that is not $K_{2}$ permits a neighbour-distinguishing 3-edge-weighting, and showed that this statement holds e.g. for the 3 -colourable graphs. It is also known, see (1), that each nice graph (i.e., a graph without a $K_{2}$-component) permits a neighbour-distinguishing 16 -edge-weighting, hence this parameter is finite.

Note that if a graph permits a neighbour-distinguishing $k$-edge-weighting, then it also permits a neighbour-distinguishing $k$-total-weighting (it is enough to put ones at all vertices), hence we obtain an upper bound $\tau(G) \leq 16$ for all graphs and $\tau(G) \leq 3$ for the 3-colourable graphs (for all graphs if the conjecture by Karoński, Łuczak and Thomason holds). Therefore, we ask if, maybe, the weights 1 and 2 are enough in the case of a total-weighting.

Conjecture 1 Every simple graph permits a neighbour-distinguishing 2-total-weighting.

\footnotetext{
${ }^{\dagger}$ The research of the first author was partially supported by MNiSW grant N N201 389134.

‡The research of the second author was partially supported by polish ministry MNiSW 
It might seem quite plausible in the face of the result by Addario-Berry, Dalal and Reed from (1), which says that for any fixed $p \in(0,1)$ the random graph $G_{n, p}$ (asymptotically) almost surely permits neighbourdistinguishing 2-edge-weighting.

In the following section we discuss the correspondence between $\chi(G)$ and $\tau(G)$. The obtained upper bound for $\tau(G)$ yields a positive verification of Conjecture 1 for the 3-colourable graphs, discussed, together with the results for the complete and 4-regular graphs, in Section 3 . The last section, Sec. 4 is devoted to decreasing the general upper bound for $\tau(G)$ from 16 down to 11 .

It is also worth mentioning here that our reasonings correspond with the recent study of Bača, Jendrol, Miller and Ryan. In (3) they introduced and studied a parameter called a total vertex irregularity strength, which is the smallest $k$ for which there exists a $k$-total-weighting such that each vertex of a graph receive a different colour, i.e. $w(u)+\sum_{e \ni u} w(e) \neq w(v)+\sum_{e \ni v} w(e)$ for each pair of vertices $u, v$ (which do not have to be adjacent). This parameter, as well as the other mentioned in this section, may be viewed as descendants of the well known irregularity strength of a graph, see (4) or (9).

Due to a considerable amount of time consumed by the reviewing process, we conclude the introduction with a short list of up-to-date results in the field. All of these were however predated and (most of them) inspired by a manuscript of this paper. First Przybyło showed in (10) that $\tau(G) \leq 7$ for all regular graphs. Then a huge break-through was a result of Kalkowski (5), who proved that $\tau(G) \leq 3$ for all graphs. Following and (considerably) modifying his algorithm led Kalkowski, Karoński and Pfender (7) to decreasing the general bound of 16 to 5 in the case of edge-weightings. Also the list version of the "1,2 Conjecture" has been introduced by Wong and Zhu (15), and independently by Przybyło and Woźniak (13). Among others one can read in either of these, that Proposition 9 of this paper can be extended to its list version. See also $(2,6$; $11,12,14)$ for more related results.

\section{Bounds for graphs with low chromatic number}

Our aim is to show that $\tau(G) \leq 2$ for a simple graph $G$. Note that $\tau(G)=1$ iff there are no neighbours with the same degree in $G$. Since we wish to distinguish only neighbours, we may assume that $G$ is a connected graph. Given a total-weighting $w$ of $G$, let $c_{w}(v):=w(v)+\sum_{e \ni v} w(e)$ (or $c(v)$ for short if the weighting $w$ is obvious) define the resulting colouring for each $v \in V(G)$. We shall call $c(v)$ a colour or a total weight of $v$. Surprisingly easily one may prove the following statement.

Proposition $2 \tau(G) \leq 2$ for bipartite graphs.

Proof: Let us first arbitrarily assign weights 1 or 2 to the edges of $G$. Then put 1 or 2 at vertices so that the resulting total weights of the vertices in one colour class are even and the total weights of the vertices in the other colour class are odd.

Consequently, $\tau(G)=2$ if $G$ is a single edge, hence our parameter makes sense for all graphs (while considering edge-weightings we must restrict ourselves to graphs without $K_{2}$-components).

Actually, similar reasoning provides us with a more general observation.

Proposition 3 Given a graph $G$ and a set of target colours $t_{v}$ for all $v \in V(G)$, there exists a 2-totalweighting $w$ of $G$ such that $c_{w}(v) \equiv t_{v}(\bmod 2)$ for each $v \in V(G)$.

In this section we discuss a generalization of this statement. Consequently, we obtain an upper bound for $\tau(G)$ based on the chromatic number of $G$. 
Lemma 4 Given a cycle $C$, a set of target colours $t_{v}$ for all $v \in V(C)$, and a positive integer $p \geq 3$, there exists $a\left(\left\lfloor\frac{p}{2}\right\rfloor+1\right)$-total-weighting $w$ of $C$ such that $c_{w}(v) \equiv t_{v}(\bmod p)$ for each $v \in V(C)$.

Proof: Let $v_{1}, \ldots, v_{n}$ be the consecutive vertices on the cycle $C,|C|=n$. We may assume $t_{v_{i}} \in[3, p+2]$ for each $i$. Let $V(C)=S \cup L$, where $v_{i} \in S$ if $t_{v_{i}} \in\left[3,\left\lfloor\frac{p}{2}\right\rfloor+2\right]$ or $v_{i} \in L$ if $t_{v_{i}} \in\left[\left\lfloor\frac{p}{2}\right\rfloor+3, p+2\right]$. Denote $t_{v_{i}}$ by $s_{i}$ if $v_{i} \in S$ or by $l_{i}$ if $v_{i} \in L$, and set $h=\left\lfloor\frac{p}{2}\right\rfloor+1$. We will use only weights $1, \ldots, h$.

Note first, that if $|L|$ is even, then we can easily find the required weighting. To do this, it is sufficient to use only numbers 1 and $h$ as the weights of the edges. We start by setting $w\left(v_{n} v_{1}\right)=1$, and then assign 1 or $h$ to the consecutive edges of $C$ one after another, so that the two edges adjacent to $v_{i}$ obtain the same weights if $v_{i} \in S$ or different ones otherwise. This way, the arities $(\bmod p)$ of the current total weights of the vertices in $S$ belong to the set $\{1,2\}$ (depending on the evenness of $p$ ) and are equal to $\left\lfloor\frac{p}{2}\right\rfloor+2$ for the ones in $L$. Hence, it is now easy to complete the weighting using numbers from the set $\{1, \ldots, h\}$ as the weights of the vertices of $C$.

Let then $|L|$ be odd and assume first that a vertex in $L$, say $v_{2}$, has the two neighbours ( $v_{1}$ and $v_{3}$ ) in $S$. If

$$
s_{1}-2+s_{3}-2 \geq l_{2}-h,
$$

then there exist $s_{1}^{\prime} \in\left[1, s_{1}-2\right], s_{3}^{\prime} \in\left[1, s_{3}-2\right]$ such that $s_{1}^{\prime}+s_{3}^{\prime}=l_{2}-h$. Set $w\left(v_{1} v_{2}\right)=s_{1}^{\prime}, w\left(v_{2}\right)=h$ and $w\left(v_{2} v_{3}\right)=s_{3}^{\prime}$ to obtain $c_{w}\left(v_{2}\right)=l_{2}$. Then set $w\left(v_{n} v_{1}\right)=w\left(v_{3} v_{4}\right)=1$ (we have $v_{n} v_{1}=v_{3} v_{4}$ if $C=C_{3}$ ). Since there remain an even number of weightless vertices in $L$, we apply the same reasoning as in the above second paragraph of the proof (by assigning weights 1 or $h$ to the remaining edges of $C$ first, and dealing with the vertices then) to obtain the desired weighting. On the other hand, if

$$
p+s_{1}-2 h+p+s_{3}-2 h \leq l_{2}-1,
$$

then there exist $s_{1}^{\prime} \in\left[p+s_{1}-2 h, h\right], s_{3}^{\prime} \in\left[p+s_{3}-2 h, h\right]$ such that $s_{1}^{\prime}+s_{3}^{\prime}=l_{2}-1$. Analogously as above, set $w\left(v_{1} v_{2}\right)=s_{1}^{\prime}, w\left(v_{2}\right)=1$ and $w\left(v_{2} v_{3}\right)=s_{3}^{\prime}$ to obtain $c_{w}\left(v_{2}\right)=l_{2}$. Then put $w\left(v_{n} v_{1}\right)=$ $w\left(v_{3} v_{4}\right)=h$ and apply the technique from the second paragraph to finish the weighting. It is easy to verify that all the weights used were in the range $[1, h]$, and that one of the inequalities (1) or 2) holds if only $h \geq 2$.

Finally, we may assume that $L$ contains two, say $v_{2}, v_{3}$, consecutive vertices of the cycle (and $|L|$ is odd). Let $l_{2} \leq l_{3}$. Then $h+l_{2}-l_{3}, l_{3}-h-1 \in\{1, \ldots, h\}$, hence it is enough to set $w\left(v_{1} v_{2}\right)=1$, $w\left(v_{2}\right)=h+l_{2}-l_{3}, w\left(v_{2} v_{3}\right)=l_{3}-h-1, w\left(v_{3}\right)=1$ and $w\left(v_{3} v_{4}\right)=h$ to obtain $c_{w}\left(v_{2}\right)=l_{2}$ and $c_{w}\left(v_{3}\right)=l_{3}$. This time we are left with an odd number of weightless vertices in $L$. However, thanks to the weights assigned to $v_{1} v_{2}$ and $v_{3} v_{4}$, we can again complete the weighting of $C$ analogously as in the second paragraph.

Lemma 5 Given a connected graph $G, u \in V(G)$, a set of target colours $t_{v}$ for all $v \in V(G)$, and a positive integer $p$, there exists $a\left(\left\lfloor\frac{p}{2}\right\rfloor+1\right)$-total-weighting $w$ of $G$ such that $c_{w}(v) \equiv t_{v}(\bmod p)$ for each $v \in V(G) \backslash\{u\}$.

Proof: Given $G$ and $u \in V(G)$, we use weights $1, \ldots,\left\lfloor\frac{p}{2}\right\rfloor+1$ to obtain the desired weighting as follows. First assign weight 1 to all the edges and vertices of $G$. If the obtained colouring does not comply with our requirements, i.e. there exists $v \in V(G) \backslash\{u\}$ with improper colour arity (not congruent to $t_{v}$ ) mod $p$, choose a path from $v$ to $u$ in $G$. By changing the weights of $v$ and an edge from this path adjacent to 
$v$, we obtain $c_{w}(v) \equiv t_{v}(\bmod p)$ for the resulting weighting $w$. Then continue traversing the path, at each step changing, if necessary, the weights of one consecutive vertex and one edge, so that at the end the colours of all the vertices from the path different from $u$ have the proper arities mod $p$. Note, we do not change the total weights of the vertices that do not belong to the path. This way we have reduced the number of the vertices in $V(G) \backslash\{u\}$ with improper colour arities. Therefore, applying the procedure described repeatedly yields the desired result.

Theorem 6 Given a connected graph $G$ which is not a tree, a set of target colours $t_{v}$ for all $v \in V(G)$, and a positive integer $p \geq 3$, there exists a $\left(\left\lfloor\frac{p}{2}\right\rfloor+1\right)$-total-weighting $w$ of $G$ such that $c_{w}(v) \equiv t_{v}(\bmod p)$ for each $v \in V(G)$.

Proof: Since $G$ is not a tree, it contains a subgraph $C$ which is a cycle. Let $u$ be an arbitrary vertex of this cycle. By Lemma 5 we may find such a weighting of $G$ that the resulting total weights of all vertices $v \in V(G) \backslash\{u\}$ are as required. Then put a temporary weight 0 at all the vertices and on each edge of $C$. Denote the resulting weighting of $G$ by $w^{\prime}$, and let $s_{v}=c_{w^{\prime}}(v)$ for $v \in V(C)$. To obtain the desired weighting, it is enough to apply Lemma 4 to $C$ with the target arities $t_{v}-s_{v}$ for each vertex $v$ of $C$.

Corollary $7 \tau(G) \leq\left\lfloor\frac{\chi(G)}{2}\right\rfloor+1$ for an arbitrary graph $G$.

Proof: By Theorem 6 (or Proposition 3 there is a $\left(\left\lfloor\frac{\chi(G)}{2}\right\rfloor+1\right.$ )-total-weighting of $G$ such that the resulting total weights have distinct arities $\bmod \chi(G)$ for vertices in different colour classes.

In the last section of this paper we show that $\tau(G) \leq 11$, see Theorem 12 , hence the above corollary provides us with a reasonable upper bound for graphs with $\chi(G) \leq 19$.

\section{Exact values of $\tau(G)$}

By Corollary 7, we obtain the following one.

Corollary 8 Each 3-colourable graph permits a neighbour-distinguishing 2-total-weighting.

The next two results exemplify the fact that $\tau(G)$ not necessarily depends on $\chi(G)$. Though the following proposition is the consequence of (3), we present here our proof for the cohesion of the article.

Proposition $9 \tau(G)=2$ for complete graphs.

Proof: We apply induction to show that it is enough to use 1 and 2 to obtain such a weighting of $K_{n}$, that the colours of its vertices are $n$ consecutive integers, either from $n$ to $2 n-1$ or from $n+1$ to $2 n$. The assertion is trivial for $n=2$.

Assume then that $n \geq 3$ and that we have found such a weighting for $K_{n-1}$. Let us add a new vertex $v$ joining it by a single edge with each vertex of $K_{n-1}$. Note that the vertices of $K_{n-1}$ carry colours from the interval $[n-1,2 n-2]$ which are consecutive integers. If the greatest of them equals $2 n-3$, we put 2 at $v$ and on all the edges incident with it. This way, the vertices of $K_{n}$ obtain $n$ different colours from the interval $[n+1,2 n]$. Analogously, if the greatest total weight at a vertex of $K_{n-1}$ equals $2 n-2$, we put 1 at $v$ and on all the edges incident with it. 
Theorem $10 \tau(G)=2$ for all 4-regular graphs.

Proof: Let $G$ be a connected 4-regular graph. If $G=K_{5}$ or $\chi(G) \leq 3$, then we are done by Proposition 9 or Corollary 8 , respectively. Hence, by Brooks' Theorem, we may assume $\chi(G)=4$. Choose the colour classes, $A, B, C$ and $D$ for $G$ so that $A$ is as large as possible, and, subject to the choice of $A, B$ is also as large as possible, and finally, subject to the choice of $A$ and $B, C$ is as large as possible as well. Consequently, each vertex in $B \cup C \cup D$ has at least one neighbour in $A$, each vertex in $C \cup D$ has at least one neighbour in $B$, and each vertex in $D$ has at least one neighbour in $C$. Let $D=D_{1} \cup D_{2}$, where $v \in D_{i}$ if $v$ has exactly $i$ neighbours in $A$, and let for $X, Y \subset V(G), E(X, Y)$ denote the set of all edges between $X$ and $Y$ in $G$. We define a weighting $w$ in the following way.

Assign 2 to the edges in $E(A, B \cup C \cup D), 1$ to the edges in $E(D, B \cup C), 2$ to the vertices in $A \cup D_{2}$, and 1 to the vertices in $D_{1}$. This way the induced subgraph $G[B \cup C]$ remains weightless, while $c(v)=10$ if $v \in A, c(v)=6$ if $v \in D_{1}$, or $c(v)=8$ if $v \in D_{2}$. For each edge $x y \in E(B, C)$ where $y \in C$, we assign 2 to it if $y$ has a neighbour in $D_{1}$ or 1 otherwise. Then choose the weights for each vertex $u$ in $B$ so that their total weights are odd. Since each of them is incident with at least one edge (in $E(B, A)$ ) with weight 2 , we have $c(u) \in\{7,9\}$. Now all adjacent vertices in $A \cup B \cup D$ are distinguished. We will put 1 or 2 at the vertices in $C$ and adjust the weights of some of the edges (in $E\left(C, D_{2}\right)$ ) and vertices (in $D_{2}$ ), so that the colours of the vertices in $A \cup B \cup D$ stay unchanged, while the vertices in $C$ are distinguished from the others. Given an arbitrary vertex $v$ in $C$, note that it is incident with at least one edge (in $E(C, A)$ ) with weight 2 and at least one with weight 1 . Consider the four edges incident with $v$.

If three of them have weight 1 , hence $N(v) \cap D_{1}=\emptyset$, we set $w(v)=1$, and thus $c(v)=6$.

If at least two of them have weight 2 and $N(v) \cap D_{2}=\emptyset$, we choose $w(v)$ so that $c(v)=8$.

Finally, if at least two of them have weight 2 and there is a vertex $y \in N(v) \cap D_{2}$, then (by our construction) $w(y)=2, w(y v)=1$ and $v$ has exactly one neighbour, say $x$, in $B$. Moreover, exactly two edges incident with $v$ have weight 1 . If $c(x)=9$, we put 1 at $v$ and get $c(v)=7$. On the other hand, if $c(x)=7$, set $w(y)=1, w(y v)=2$ and $w(v)=2$. This way the colour of $y$ remains unchanged, while $c(v)=9$.

After processing all the vertices $v$ in $C$ we obtain a weighting that is neighbour-distinguishing.

\section{General bound}

In (1), the following theorem was proved.

Theorem 11 Let $G=(V, E)$ be a bipartite graph with bipartition $V=X \cup Y$. For each $v \in X$, let $a_{v}^{-}=\left\lfloor\frac{d(v)}{2}\right\rfloor$ and set $a_{v}^{+}=a_{v}^{-}+1$. For each $v \in Y$, fix any $a_{v}^{-}$, $a_{v}^{+}$such that $a_{v}^{-} \leq\left\lfloor\frac{d(v)}{2}\right\rfloor \leq a_{v}^{+}$and

$$
a_{v}^{+} \leq \min \left(\frac{d(v)+a_{v}^{-}}{2}+1,2 a_{v}^{-}+1\right) .
$$

Then there is a spanning subgraph $H$ of $G$ such that $d_{H}(v) \in\left\{a_{v}^{-}, a_{v}^{+}\right\}$for all $v \in V$.

We use it, together with the construction by Addario-Berry, Dalal and Reed from (1), to prove the following statement.

Theorem 12 Every graph permits a neighbour-distinguishing 11-total-weighting. 
Proof: Let $G$ be a connected graph. For any ordering of a set of its vertices, let $F\left(v_{i}\right)=\left\{v_{j} \mid v_{j} \in\right.$ $N\left(v_{i}\right)$ and $\left.j>i\right\}$ and $B\left(v_{i}\right)=\left\{v_{j} \mid v_{j} \in N\left(v_{i}\right)\right.$ and $\left.j<i\right\}$. Call these sets the forward and the backward neighbours of $v_{i}$, respectively. Choose an ordering of $V(G)$ that maximizes $k=\max \{j$ : $\left|F\left(v_{i}\right)\right|>\left|B\left(v_{i}\right)\right|$ for $\left.i \leq j\right\}$. Put the first $k$ vertices in $V_{1}$ and the remaining ones into a temporary set $T$. Note that $k$ defined above does not decrease regardless of any reordering of the vertices within $T$. Moreover, $d_{T}(v) \leq d_{V_{1}}(v)$ for all $v \in T$. (Otherwise, we could move $v$ to the $(k+1)$ 'st position of the ordering and thereby create an ordering with a larger value of $k$.)

Next, apply the above described procedure to the induced graph $G[T]$, to find a set $V_{2} \subseteq T$ and a new ordering of the vertices in $T$. Remove the vertices of $V_{2}$ from the temporary set $T$. Then repeat this procedure twice more to find $V_{3}, V_{4}$ and the final ordering of the vertices of $G$. Let $V_{5}=V(G) \backslash\left(V_{1} \cup\right.$ $\left.V_{2} \cup V_{3} \cup V_{4}\right)$. Note that each $v \in V_{i}, i=1,2,3,4$, has strictly fewer backward neighbours in $V_{i}$ than forward neighbours. By the observation at the end of the previous paragraph, for each $v \in V_{5} \subset T$, $d_{V_{2}}(v)+d_{V_{3}}(v)+d_{V_{4}}(v)+d_{V_{5}}(v)=d_{V_{2} \cup V_{3} \cup V_{4} \cup V_{5}}(v) \leq d_{V_{1}}(v)$. Analogously we have $d_{V_{3}}(v)+$ $d_{V_{4}}(v)+d_{V_{5}}(v) \leq d_{V_{2}}(v), d_{V_{4}}(v)+d_{V_{5}}(v) \leq d_{V_{3}}(v), d_{V_{5}}(v) \leq d_{V_{4}}(v)$, and hence $8 d_{V_{5}}(v) \leq d_{V_{1}}(v)$ for each $v \in V_{5}$.

Consider the edges from $V_{5}$ to $V_{1}$. Since every vertex $v \in V_{5}$ has at least $8 d_{V_{5}}(v)$ edges to $V_{1}$, we can choose a subset (of these edges) where each $v \in V_{5}$ has exactly $8 d_{V_{5}}(v)$ edges to $V_{1}$. Let $B$ be the bipartite graph spanned by this reduced set of edges.

Now set $w(e)=2$ for each edge of $G$. Then assign weights between 3 and 10 to the vertices so that the resulting colour of each vertex has an arity mod 8 as specified in Table 1 . We will modify this weighting

\begin{tabular}{|c|c|c|c|c|}
\hline$V_{1}$ & $V_{2}$ & $V_{3}$ & $V_{4}$ & $V_{5}$ \\
\hline 1 & 3 & 5 & 7 & $0,2,4$ or 6 \\
\hline
\end{tabular}

Tab. 1: Target arity for partition elements

maintaining the specified colour arities mod 8 and eliminating possible internal conflicts within the sets $V_{1}, \ldots, V_{5}$.

Process the vertices of $V_{1} \cup V_{2} \cup V_{3} \cup V_{4}$ one after another according to the specified order. Given a vertex $v \in V_{i}$, we add 8 to the weights of an arbitrary subset of the forward edges of $v$ in order to distinguish its colour from the colours of its backward neighbours in $V_{i}$. This is always feasible, since $v$ has at least one more forward neighbour (not necessarily in $V_{i}$ ) than backward neighbours in $V_{i}$.

After processing all the vertices in $V_{1} \cup V_{2} \cup V_{3} \cup V_{4}, w(e) \in\{2,10\}, 3 \leq w(v) \leq 10$ for each edge $e$ and each vertex $v$ of $G$, while the arities $(\bmod 8)$ of colours are as specified in Table 1 . Moreover, every two neighbours from $V_{1} \cup V_{2} \cup V_{3} \cup V_{4}$ have distinct colours. In our final step, we adjust the weights of edges in $B$ to distinguish adjacent vertices in $V_{5}$. First we use Theorem 11 where $X=V_{1} \cap V(B)$ and $Y=V_{5} \cap V(B)$ to determine a subgraph $H$ of $B$. Then for each edge $e$ of $B$ we will add 1 to its weight if $e \in E(H)$ or subtract 1 otherwise (we may do so, since $w(e) \in\{2,10\}$ ). At the end we modify the weights of the vertices in $V_{1}$ so that their colours are as they were before this final step.

Define $\left\{a_{v}^{-}, a_{v}^{+}\right\}$for each $v \in X$ by setting $a_{v}^{-}=\left\lfloor\frac{d_{B}(v)}{2}\right\rfloor$ and $a_{v}^{+}=a_{v}^{-}+1$. Then choose $\left\{a_{v}^{-}, a_{v}^{+}\right\}$ for each vertex in $Y$ as follows. Process the vertices of $Y$ in any order. For each $v \in Y$ in turn, we choose $a_{v}^{-} \in\left[\frac{d_{B}(v)}{4}, \frac{d_{B}(v)}{2}\right]$ (this range has integer endpoints, because 8 divides $d_{B}(v)$ ), and set $a_{v}^{+}=a_{v}^{-}+$ $\frac{d_{B}(v)}{4}+1$. We make our choice to ensure that for any previously processed neighbour $u \in V_{5}$ of $v$, for any 
$a_{v} \in\left\{a_{v}^{-}, a_{v}^{+}\right\}$, and for any $a_{u} \in\left\{a_{u}^{-}, a_{u}^{+}\right\}$, we have $c(v)+a_{v}-\left(d_{B}(v)-a_{v}\right) \neq c(u)+a_{u}-\left(d_{B}(u)-a_{u}\right)$, where by $c(v), c(u)$ we mean the current colours of $v, u$ (and $c(v)+a_{v}-\left(d_{B}(v)-a_{v}\right)$ will be the colour of $v$ after the final step.) This is possible since each previously processed neighbour of $v$ can prevent at most two choices for $a_{v}^{-}$and there are precisely $2 d_{V_{5}}(v)+1$ choices available for $a_{v}^{-}$.

This set of degree choices satisfies the conditions of Theorem 11. Indeed, it is clear for the vertices in $X$. Also obviously $a_{v}^{-} \leq\left\lfloor\frac{d_{B}(v)}{2}\right\rfloor \leq a_{v}^{+}$for each $v \in Y$, so it remains to show that for all $v \in Y$, (3) holds. Since $a_{v}^{-} \leq \frac{d_{B}(v)}{2}$, then $a_{v}^{+}=a_{v}^{-}+\frac{d_{B}(v)}{4}+1=\frac{d_{B}(v)}{4}+\frac{a_{v}^{-}}{2}+\frac{a_{v}^{-}}{2}+1 \leq \frac{d_{B}(v)}{2}+\frac{a_{v}^{-}}{2}+1$.

On the other hand, since $a_{v}^{-} \geq \frac{d_{B}(v)}{4}$, then $a_{v}^{+}=a_{v}^{-}+\frac{d_{B}(v)}{4}+1 \leq 2 a_{v}^{-}+1$. Thus, by Theorem 11 . a subgraph $H$ of $B$ exists such that after performing the previously described additions and subtractions on the set of edges of $B$, all adjacent vertices in $V_{5}$ have distinct colours. Note that such changes may influence the total weights of the vertices in $V_{1}$, adding 1 or 2 to some of them, or decreasing them by 1 (depending on the evenness of $d_{B}(v)$ and the choice of $a_{v}^{-}$or $a_{v}^{+}$). Then however, for each such $v \in V_{1}$ (since $3 \leq w(v) \leq 10$ ) we balance this change by subtracting 1 or 2 , or by adding 1 , respectively, to its weight $w(v)$.

Note that the arities $(\bmod 8)$ of colours of the vertices in $V_{5}$ remain as they are specified in Table 1 (they are even numbers), and that $w(v), w(e) \in\{1, \ldots, 11\}$ for all $v \in V(G)$ and all $e \in E(G)$, hence the described construction provides us with a neighbour-distinguishing 11-total-weighting.

\section{References}

[1] L. Addario-Berry, K. Dalal, B. A. Reed, Degree constrained subgraphs, Proceedings of GRACO2005, volume 19 of Electron. Notes Discrete Math., Amsterdam (2005), 257-263 (electronic), Elsevier.

[2] M. Anholcer, M. Kalkowski, J. Przybyło, A new upper bound for the total vertex irregularity strength of graphs, Discrete Math. 309 (21) (2009) 6316-6317.

[3] M. Bača, S. Jendrol̆, M. Miller, J. Ryan, On Irregular Total Labelings, Discrete Math. 307 (2007) 1378-1388.

[4] G. Chartrand, M. S. Jacobson, J. Lehel, O. R. Oellermann, S. Ruiz, F. Saba, Irregular networks, Proc. of the 250th Anniversary Conf. on Graph Theory, Fort Wayne, Indiana (1986).

[5] M. Kalkowski, On 1,2-conjecture, submitted.

[6] M. Kalkowski, M. Karoński, F. Pfender, A new upper bound for the irregularity strength of graphs, manuscript.

[7] M. Kalkowski, M. Karoński, F. Pfender, Vertex-coloring edge-weightings: Towards the 1-2-3conjecture, to appear in J. Combin. Theory Ser. B.

[8] M. Karoński, T. Łuczak, A. Thomason, Edge weights and vertex colours, J. Combin. Theory, Ser. B 91 (2004) 151-157.

[9] J. Lehel, Facts and quests on degree irregular assignments, Graph Theory, Combinatorics and Applications, Willey, New York, (1991) 765-782. 
[10] J. Przybyło, A note on a neighbour-distinguishing regular graphs total-weighting, Electron. J. Combin. 15 (1) (2008) $\sharp N 35$.

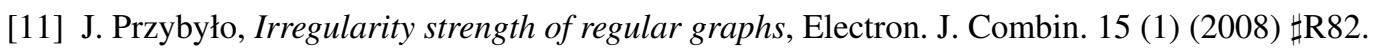

[12] J. Przybyło, Linear bound on the irregularity strength and the total vertex irregularity strength of graphs, SIAM J. Discrete Math. 23 (1) (2009) 511-516.

[13] J. Przybyło, M. Woźniak, Total weight choosability of graphs, submitted.

[14] T. Wong, D. Yang, X. Zhu, Total weighting of graphs by max-min method, submitted.

[15] T. Wong, X. Zhu, Total weight choosability of graphs, submitted. 